\title{
Thermoplastic High Performance Cable Insulation Systems for Flexible System Operation
}

\author{
A. S.Vaughan, C. D. Green and I. L. Hosier \\ ECS, University of Southampton \\ Southampton, SO17 1BJ UK
}

\author{
S. J. Sutton \\ Consultant \\ Egham, Middx. UK
}

\author{
G. C. Stevens, A. Pye and J. L. Thomas \\ GnoSys Global Ltd, \\ Guildford, GU2 7YD, UK
}

\author{
T. Geussens \\ Dow Europe GmbH \\ CH-8810 Horgen, $\mathrm{CH}$
}

\begin{abstract}
Crosslinked polyethylene (XLPE) has been the cable insulation material of choice in many different transmission and distribution applications for many years and, while this material has many desirable characteristics, its thermomechanical properties have consequences for both continuous and emergency cable ratings which, in turn, have implications for system operational flexibility. In this paper, we describe the principles and two embodiments through which new thermoplastic insulation systems can be actively designed with improved electrical and thermo-mechanical properties for use in cable applications. First, a blend system based upon high density (HDPE) and low density polyethylene (LDPE) is considered, before comparable principles are applied to combinations of polypropylene grades. In both cases, a suitable formulation is first developed through laboratory testing of film and plaque specimens, before a mini-cable is produced and tested.
\end{abstract}

Keywords-cable; polyethylene; polypropylene; blend; dielectric breakdown; thermo-mechanical properties

\section{INTRODUCTION}

Crosslinked low density polyethylene (XLPE) exhibits a desirable combination of properties for use as the dielectric in cable systems and, consequently, is now widely used in this application. However, environmental concerns have led both to XLPE recycling technologies [1] and to increasing interest in the use of novel thermoplastic materials [2], e.g. Prysmian's PLaser technology. In addition to recyclability, the use of thermoplastics also brings further benefits. Cables can be manufactured more quickly in a one-shot process with no crosslinking; higher material purities can be reached through the use of finer melt filters since, in thermoplastics, there is no risk of amber generation due to premature crosslinking; there will be no crosslinking by-products.

However, in general, single polymers do not exhibit an appropriate combination of properties for use in a cable, which would ideally include, amongst others, high breakdown strength, low temperature flexibility and good mechanical integrity at high temperatures. This final factor is particularly

The authors acknowledge the support of the Technology Strategy Board, the Engineering and Physical Sciences Research Council, Dow Electrical and Telecommunications and National Grid. significant, since it is closely related to the rating of the cable under both steady state and emergency conditions; XLPE melts at around $90{ }^{\circ} \mathrm{C}$, which limits such factors [3].

The work described here concerns the development of two potential new cable insulation technologies based upon thermoplastic blends, in which properties are optimised by combining different polymers. The overarching rationale behind this strategy is that thermoplastics are both greener (end of life recycling; reduced energy consumption and emissions during manufacture) and offer in-service operational benefits compared with XLPE. In particular, emergency ratings are dramatically improved. In both of the technologies presented here, molecular composition is first optimised in the laboratory, based upon morphological evolution, electrical breakdown behaviour and mechanical factors. Then, the work goes on to address two critical issues, namely: is it possible to extrude such a system to give a cable and does the laboratoryoptimised material still exhibit desirable characteristics when extruded onto a cable?

\section{EXPERIMENTAL}

\section{A. Polyethylene Materials}

The high density polyethylene (HDPE) used in this study was Dow 40055E and the low density polyethylene (LDPE) was a non-commercial Dow material used as the base resin in certain cable-grade insulation products. Since the required blend composition (20\% HDPE: $80 \%$ LDPE) had already been established in previous isothermal work [4], pellets of precompounded blend for small-scale laboratory testing were prepared using a Haake PTW 16/40D twin screw lab extruder equipped with a $3 \mathrm{~mm}$ capillary die and pelletizer. Large-scale pellet production for mini-cable extrusion was undertaken on a Berstorff ZE40UT twin screw extruder. The LDPE and XLPE reference mini-cables were produced using the same LDPE base as in the blend.

\section{B. Polypropylene Materials}

The isotactic polypropylene (iPP) used in this study was the Dow H358-02 system, which was combined with one of three propylene-ethylene copolymer (PEC) systems (VERSIFYTM 
2200, 2300 and 2400) which, respectively, contain 9, 12 and $15 \mathrm{~mol} \%$ of ethylene. Blends were prepared in the laboratory using both a standard solution method, involving dissolution of the required components in xylene, and by melt-mixing using a Brabender Plastograph with a W50EHT mixing attachment. The former approach is more suitable for the preparation of small volumes of a large number of different formulations; the latter is more technologically relevant. Material preparation for mini-cable production was undertaken using the same equipment as in the PE case described above. Throughout this paper, the various PP blend systems are designated as follows: $(\mathrm{S} / \mathrm{M} / \mathrm{E})(2200 / 2300 / 2400)(50 / 37.5)$, where the first letter indicates solution blending $(\mathrm{S})$, melt blending $(\mathrm{M})$ or extrusion (E), the middle number indicates the member of the VERSIFY' ${ }^{\mathrm{TM}}$ series used in the blend and the final number defines the percentage iPP by weight percent (wt\%). In figures where composition constitutes the independent variable, the final number is omitted.

\section{Sample preparation}

Film samples ( $\sim 85 \mu \mathrm{m}$ in thickness) for breakdown testing and plaques $(\sim 1.7 \mathrm{~mm}$ in thickness $)$ for mechanical characterisation were pressed using an appropriate hydraulic press and the required cooling history was then imposed using a Mettler Toledo FP82 hotstage. Complete details of these procedures are provided elsewhere $[5,6]$. Mini-cable, with a nominal insulation thickness of $4 \mathrm{~mm}$, was manufactured on a Troester triple extrusion line using a 7-stranded aluminium conductor of cross-sectional area $25 \mathrm{~mm}^{2}$. In all cases, an inner semiconducting (semicon) screen was extruded along with the insulation but, to facilitate subsequent $\mathrm{HV}$ testing, no outer semicon was extruded.

\section{Material Charactisation}

Breakdown of the above thin film specimens was conducted using our standard AC ramp testing methodology. Samples were inserted into a specially designed testing chamber containing Dow Corning 200/20cs silicone fluid. A sinusoidal $50 \mathrm{~Hz}$ voltage was applied to the sample with a peak-peak amplitude ramp of $141 \mathrm{~V} \mathrm{~s}^{-1} \pm 4 \%$ until breakdown. The thickness of the specimen was subsequently measured at each breakdown site such that the associated field, $E$, at breakdown could be determined. The resulting datasets were analysed assuming a 2-parameter Weibull distribution. Scale and shape parameters were calculated using the maximum likelihood estimation technique in the Reliasoft Weibull ${ }^{++} 7$ software and likelihood ratios were used to generate $90 \%$ confidence intervals.

The temperature dependence of the mechanical modulus was determined using a Rheometrics RSAII dynamic mechanical thermal analysis (DMTA) system operating in dual cantilever mode at a constant frequency of $1 \mathrm{~Hz}$.

Structural studies were undertaken using a JEOL JSM-5910 scanning electron microscope (SEM). The required samples were first cut open using an RMC CR21/MT7 cryoultramicrotome before being etched for $2 \mathrm{~h}$ with a permanganic

TM: Trademark of The Dow Chemical Company ("Dow") or an affiliated company of Dow. reagent, using standard procedures [7].

\section{E. Cable Testing}

It proved impractical to determine the AC breakdown strength of the mini-cables and, consequently, DC testing was undertaken at room temperature using a Henry Patterson \&

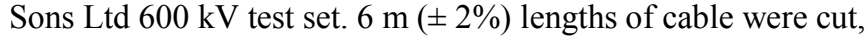
crimped to form a loop and hung from a $3 \mathrm{~m}$ fiber-glass rod attached to a winch. The conductor was connected to the HVDC supply using a $2 \mathrm{~m}$ copper pipe; a trough of tap water formed the ground electrode. A voltage ramp was then applied to each specimen in a stepwise fashion: $1.75 \mathrm{kV} \mathrm{s}^{-1}+/-13 \%$ rises for $8 \mathrm{~s}$, followed by $30 \mathrm{~s}$ dwells, in order to give an overall average ramp rate of $370 \mathrm{~V} \mathrm{~s}^{-1}+/-7 \%$. For safety reasons, it was not possible to apply a voltage greater than $400 \mathrm{kV}$ to the mini-cables.

\section{RESUlTS}

\section{A. Polyethylene Blend Systems}

For HDPE/LDPE blends of the composition specified above, an isothermal crystallization temperature $\left(T_{c}\right)$ window $\left(113-119^{\circ} \mathrm{C}\right)$ exists in which material with enhanced properties are formed [5]. These properties stem from a twostage crystallization process that generates a space-filling array of lamellar crystals composed primarily of HDPE; such a morphology is shown in Fig. 1. Since prolonged isothermal crystallization is technologically impractical, this study set out, initially, to identify a cooling range within which comparable effects could be produced. Fig. 2 shows breakdown data presented in the form of Weibull contour plots $(90 \%$ confidence level) that indicate that any cooling rate within at least the range $0.5{ }^{\circ} \mathrm{C} \mathrm{min}{ }^{-1}$ to $10{ }^{\circ} \mathrm{C} \mathrm{min}^{-1}$ will generate specimens with a morphology that conveys an increased breakdown strength on the resulting material. That is, a wide range of cooling rates can reproduce the electrical effects initially reported to result from isothermal crystallization at intermediate temperatures [4].

Fig. 3 presents mechanical data obtained from differently processed samples of the PE blend and, for comparison, XLPE.

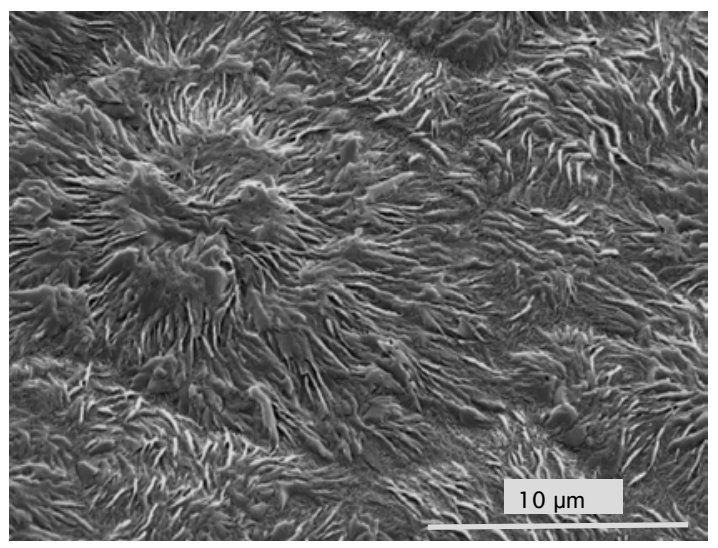

Fig. 1. SEM image showing the continuous space filling morphology of a $20 \%$ HDPE: $80 \%$ LDPE blend that typifies isothermal crystallization within the temperature range $113-119^{\circ} \mathrm{C}$. 


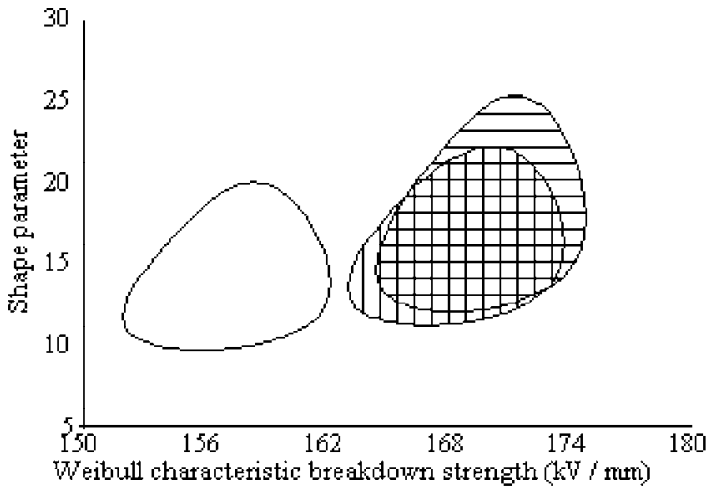

Fig. 2. Weibull contour plots representing sections of the likelihood surface at a $90 \%$ level derived from breakdon data obtained from PE blend samples prepared by quenching (open contour), cooling at $10{ }^{\circ} \mathrm{C} \mathrm{min}^{-1}$. (vertical fill) and $0.5{ }^{\circ} \mathrm{C} \min ^{-1}$ (horizontal fill).

From this, it is evident that, at low temperatures, both the quenched and slow cooled blends are slightly stiffer than the XLPE reference. However, particularly in the case of the slow cooled blend, mechanical integrity is retained to much higher temperatures, implying that a cable constructed using such a blend could accommodate an operating temperature some $30{ }^{\circ} \mathrm{C}$ higher than XLPE, at least for a relatively short period of time.

\section{B. Polypropylene Blend Systems}

In terms of both structure and properties, the broad principles described above for polyethylene blends also apply to polypropylene blends, in that continuous space-filling morphologies lead to desirable properties. However, in the case of iPP/PEC blends, the precise molecular architecture of the copolymer exerts a dramatic influence on behaviour, whereas the choice of LDPE in the polyethylene case is of secondary importance. Fig. 4 shows the effect of both composition and choice of PEC on the breakdown strength (i.e. the maximum likelihood Weibull scale parameter) of a selection of PP blends. In view of the results presented previously, all of these were cooled slowly, at $1{ }^{\circ} \mathrm{C} \mathrm{min}^{-1}$. From this, an optimal composition of $50 \mathrm{wt} \%$ iPP is apparent for all blends when breakdown testing is conducted at either $30^{\circ} \mathrm{C}$ or $120^{\circ} \mathrm{C}$.

From an electrical breakdown viewpoint, the system containing $50 \%$ iPP and 50\% VERSIFY ${ }^{\mathrm{TM}} 2200$ (i.e. M2200/50) appears close to optimal, as far as the various material combinations considered in this study are concerned. However, any practically viable cable material must also exhibit reasonable low temperature flexibility and high temperature thermo-mechanical integrity. Fig. 5 compares the temperature dependence of the dynamic modulus of samples of M2200/50 crystallised at two different cooling rates with the behavior of the XLPE reference system. From this, it is evident that all the systems considered here are comparable in terms of their low temperature flexibility and, indeed, the low temperature performance of the PP blend is somewhat better than that of the PE blend shown in Fig. 3. Clearly, the high temperature integrity of M2200/50 is better than that of the PE blend and very much better than XLPE, as would be anticipated. Comparison of these results with equivalent data obtained from the other systems shown in Fig. 4 demonstrates

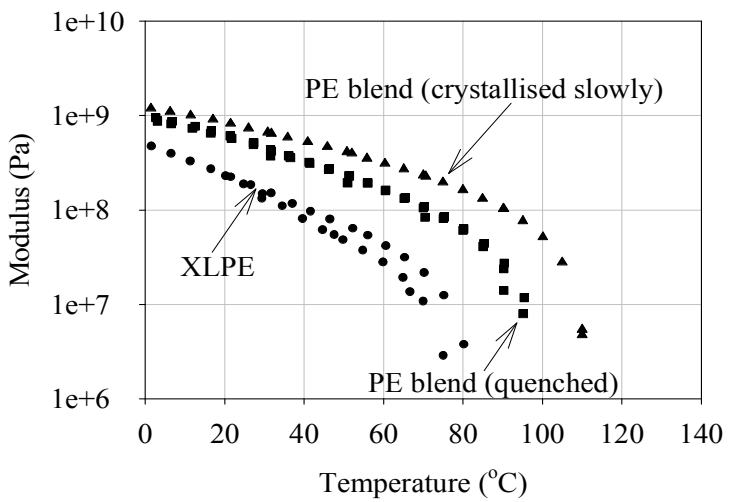

Fig. 3. DMTA data obtained from the PE blend (quenched and crystallised slowly at $1{ }^{\circ} \mathrm{C} \mathrm{min}^{-1}$ ) and an XLPE reference.

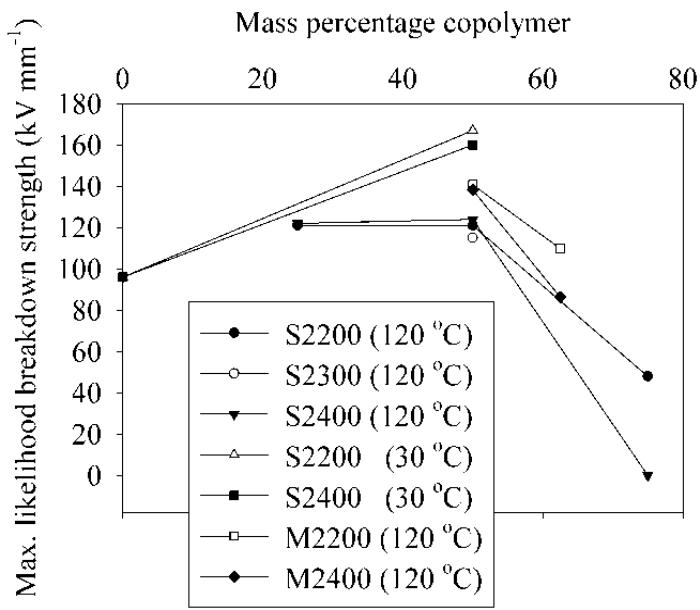

Fig. 4. Effect of composition and testing temperature on breakdown strength for solution and melt-mixed PP blends crystallized at $1{ }^{\circ} \mathrm{C} \mathrm{min}-1$.

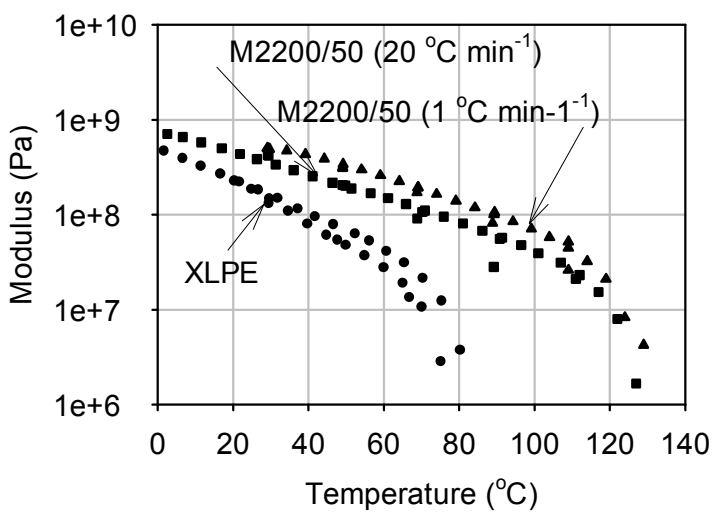

Fig. 5. DMTA data for the M2200/50 PP blend (crystallized at $20{ }^{\circ} \mathrm{C} \mathrm{min}^{-1}$ and $1{ }^{\circ} \mathrm{C} \mathrm{min}{ }^{-1}$ ) and an XLPE reference.

that none of these exhibit significant mechanical benefits compared with M2200/50 [6]. Consequently, we conclude that when electrical and mechanical characteristics are considered in concert, out of the various base polymers considered here, a combination of 50\% iPP and 50\% VERSIFYTM 2200 would be most appropriate for mini-cable production. 


\section{Cable Testing}

Fig. 6 contains two SEM micrographs showing typical cross-sections through the mini-cables extruded using (a) the designed PE blend and (b) the designed PP blend (E2200/50). Unsurprisingly, the two systems exhibit somewhat different morphologies but, in line with the principles presented in connection with Fig. 1, both are continuous and fine scaled and in neither is there evidence of extensive phase separation or potentially weak boundary regions between adjacent structural elements. As such, the combination of material composition, extrusion conditions and cooling rate used in manufacture has, from a structural perspective, met our objectives.
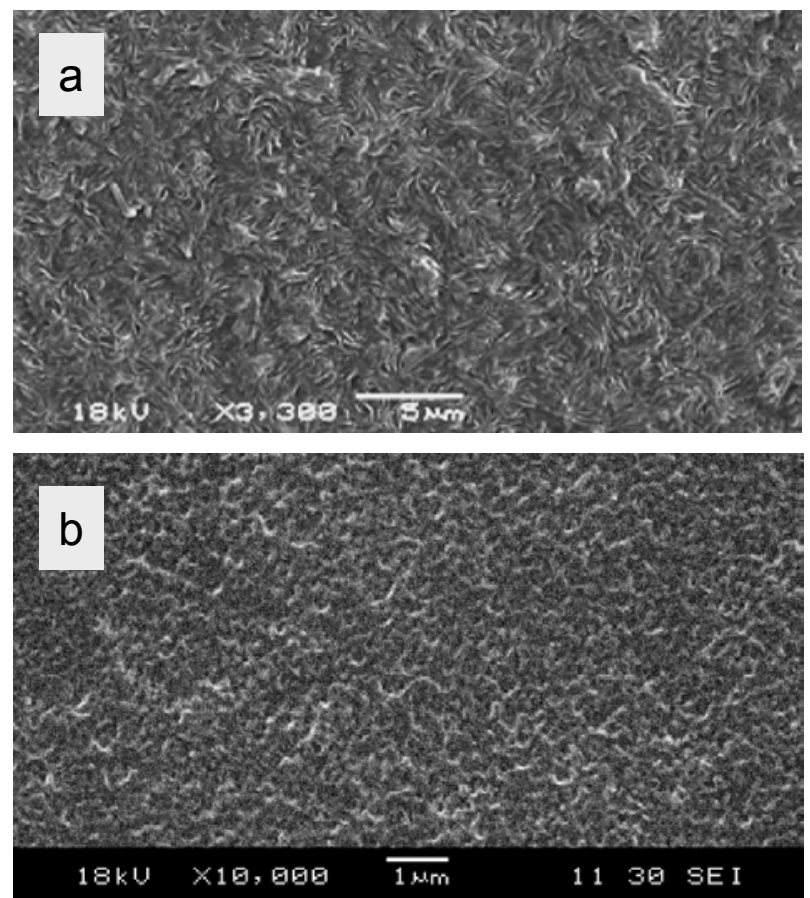

Fig. 6. SEM micrographs showing typical mini-cable morphologies for insulation composed of (a) 20\% HDPE and $80 \%$ LDPE and (b) 50\% iPP and $50 \%$ VERSIFY $^{\mathrm{TM}} 2200$. In both cases, the morphology is continuous with a fine scale texture.

Table 1 contains breakdown data obtained from the minicables manufactured using the designed blends and, for comparison, a reference XLPE cable produced under equivalent conditions and tested in an identical manner. In the case of the XLPE, all the specimens that were tested failed before the maximum voltage of $400 \mathrm{kV}$ was reached; conversely, none of the extruded thermoplastic systems failed. While this result is disappointing in that we are therefore unable to determine the extent of the improved performance for either blend compared with XLPE, these results do indicate that, electrically, both blend systems are extremely good when extruded onto a cable. Indeed, when the actual insulation thickness is also considered, the performance of the cable insulated with the PP blend is even more impressive, since a statistical analysis of the insulation thickness in the mini-cables
TABLE I. Mini-CABLE BREAKDOWN Voltage DATA

\begin{tabular}{|c|c|c|c|c|c|}
\hline \multirow{2}{*}{$\begin{array}{c}\text { Insulation } \\
\text { System }\end{array}$} & \multicolumn{5}{|c|}{ Breakdown Voltage (kV) } \\
\cline { 2 - 6 } & $\begin{array}{c}\text { Sample } \\
\text { No. 1 }\end{array}$ & $\begin{array}{c}\text { Sample } \\
\text { No. 2 }\end{array}$ & $\begin{array}{c}\text { Sample } \\
\text { No. 3 }\end{array}$ & $\begin{array}{c}\text { Sample } \\
\text { No. 4 }\end{array}$ & $\begin{array}{c}\text { Sample } \\
\text { No. 5 }\end{array}$ \\
\hline PE Blend & $>400$ & $>400$ & $>400$ & $>400$ & $>400$ \\
\hline PP Blend & $>400$ & $>400$ & $>400$ & $>400$ & $>400$ \\
\hline XLPE reference & 184 & 168 & 224 & 196 & 196 \\
\hline
\end{tabular}

insulated with E2200/50 and XLPE indicated an average insulation thickness of $3.39 \mathrm{~mm}$ and $4.34 \mathrm{~mm}$ respectively. The electrical superiority of the PP-based blend is, therefore, even greater than implied by Table 1 .

\section{CONCLUSIONS}

This study set out to consider the potential of two different designed polymer blend systems for use as next generation cable insulation materials. In both cases, the design was motivated by the desire to develop insulation materials with green credentials that, in service, could offer increased operational flexibility as a consequence of their improved high temperature integrity. Based on laboratory testing, one PE blend and one PP blend were identified and used to manufacture mini-cables; an XLPE-insulated mini-cable was also produced, to act as a reference. Despite a lack of experience of how to extrude thermoplastics, the use of an XLPE extrusion line in manufacture and the incorporation of non-optimised semicons, both thermoplastic-insulated minicables still outperformed the XLPE-insulated, and much more optimized, analogue.

\section{REFERENCES}

[1] Y. Ohki, "XLPE recycling technology in Japan", IEEE Electr. Insul. Mag., vol. 25, pp. 48-49, Mar-Apr. 2009.

[2] L. Castellani, R.E. Grizante, A. Zaopo and E. Albizzati, Pirelli Cavi e Sistemi S.P.A., US patent US 6410651 (B1), 2002.

[3] J. A. Pilgrim, P. L. Lewin and A. S. Vaughan, "Quantifying the operational benefits of new HV cable systems in terms of dielectric design parameters" Proc. 2012 IEEE International Symposium on Electrical Insulation, pp. 261-265, Jun. 2012.

[4] I. L. Hosier, A. S. Vaughan, and S. G. Swingler, "Structure-property relationships in polyethylene blends: the effect of morphology on electrical breakdown strength", J. Mater. Sci., vol. 32, pp. 4523-4531, Sept. 1997.

[5] C. D. Green, A. S. Vaughan, G. C. Stevens, S. J. Sutton and T. Geussens, "Recyclable power cable comprising a blend of slowcrystallized polyethylenes", IEEE Trans. Diel. Electr. Insul., vol. 20, pp.1-9, Feb. 2013.

[6] C. D. Green, et al., "Thermoplastic cable insulation comprising a blend of isotactic polypropylene and a propylene-ethylene copolymer", IEEE Trans. Diel. Electr. Insul., in press.

[7] R. H. Olley and D. C. Bassett, "An improved permanganic etchant for polyolefins", Polymer, vol. 23, pp. 1707-1710, Nov. 1982. 